\title{
Is the problem of molecular structure just the quantum measurement problem?
}

Sebastian Fortin and Olimpia Lombardi

CONICET - Universidad de Buenos Aires

\begin{abstract}
In a recent article entitled "The problem of molecular structure just is the measurement problem", Alexander Franklin and Vanessa Seifert argue that insofar as the quantum measurement problem is solved, the problems of molecular structure are resolved as well. The purpose of the present article is to show that such a claim is too optimistic. Although the solution of the quantum measurement problem is relevant to how the problem of molecular structure is faced, such a solution is not sufficient to account for the structure of molecules as understood in the field of chemistry.
\end{abstract}




\title{
Is the problem of molecular structure just the quantum measurement problem?
}

\author{
Sebastian Fortin and Olimpia Lombardi
}

CONICET - Universidad de Buenos Aires

\section{Introduction}

One of the hottest topics in the philosophy of chemistry is the relation between chemistry and physics. In this context, the central role is played by the problem of molecular structure, consisting in the fact that the spatial features of the molecule find no comfortable place in the theoretical framework of quantum mechanics. Some authors seem to retain an ontologically reductionistic view by conceiving the problem as the result of deep epistemic limitations (Sutcliffe and Woolley 2011, 2012a) or of an inadequate formulation of the issue (Hettema 2012). Others consider that quantum decoherence supply the reduction link between classical molecular chemistry and the quantum realm (Trost and Hornberger 2009, Scerri 2011, 2013). From a different view, it has been claimed that molecular structure cannot be strictly explained by quantum mechanics (Primas 1983, 1998, Amann 1992, Martínez González et al 2019), even when decoherence is taken into account (Fortin et al. 2106). Nevertheless, this limitation is not always interpreted in the same way. For some authors, the impossibility of strict explanation is a manifestation of the merely approximate character of the concept of molecular structure when confronted with the precise quantum description (Woolley 1978, 1982, Bader 1990, 2011). Others, by contrast, take an ontologically non-reductionist stance and conceive the case of molecular structure as an example of ontological emergence (Hendry 1998, 2004, 2008, 2010, Matta et al. 2020).

In a recent article entitled "The problem of molecular structure just is the measurement problem", Alexander Franklin and Vanessa Seifert (2020) consider the problem of molecular structure from a different perspective. By analyzing three particular manifestations of the issue, they "argue that these problems are just special cases of the measurement problem of quantum mechanics: insofar as the measurement problem is solved, the problems of molecular structure are resolved as well" (2020, Abstract). The purpose of the present paper is to show that such a claim is too optimistic. Of course, the solution of the quantum measurement problem is highly relevant to how the problem of molecular structure is faced. However, the mere solution of the measurement problem is not sufficient to account for the particular geometrical features of the molecule. In order to argue in this sense, the article is 
organized as follows. In Section 2, the problem of quantum measurement will be revisited. Then, the three problems as presented in Franklin and Seifert's paper will be considered: enantiomers and Hund's paradox (Section 3), isomers and the inability of resultant Hamiltonians to determine molecular structure (Section 4), and symmetry breaking (Section 5). Some final remarks (Section 6) will close the argumentation.

\section{The quantum measurement problem}

As it is well known, the measurement problem is the most discussed topic in the debates about the interpretation of quantum mechanics. According to the standard von Neumann model (see, e.g., Myrvold 2018), a quantum measurement is conceived as an interaction between a system $S$, corresponding to the Hilbert space $\mathcal{H}_{S}$, and a measuring apparatus $M$, corresponding to the Hilbert space $\mathcal{H}_{M}$. Before the interaction, $M$ is prepared in a ready-tomeasure state $\left|p_{0}\right\rangle \in \mathcal{H}_{M}$, eigenstate of the pointer observable $\hat{P}$ of $M$. The apparatus is constructed in such a way that the interaction establishes a correlation between the eigenstates $\left|a_{i}\right\rangle \in \mathcal{H}_{S}$ of an observable $\hat{A}$ of $S$ and the eigenstates $\left|p_{i}\right\rangle \in \mathcal{H}_{M}$ of the observable $\hat{P}$ of $M$ :

$$
\left|a_{i}\right\rangle \otimes\left|p_{0}\right\rangle \rightarrow\left|a_{i}\right\rangle \otimes\left|p_{i}\right\rangle
$$

Now let us suppose that the system $S$ is prepared in a superposition of the eigenstates of $\hat{A}$, $\sum_{i} c_{i}\left|a_{i}\right\rangle$, where at least two of the $c_{i}$ are nonzero. If the evolution of this state is the linear Schrödinger evolution, the resulting process is

$$
\left|\psi_{0}\right\rangle=\sum_{i} c_{i}\left|a_{i}\right\rangle \otimes\left|p_{0}\right\rangle \rightarrow\left|\psi_{1}\right\rangle=\sum_{i} c_{i}\left|a_{i}\right\rangle \otimes\left|p_{i}\right\rangle,
$$

with $\left|\psi_{0}\right\rangle,\left|\psi_{1}\right\rangle \in \mathcal{H}_{S} \otimes \mathcal{H}_{M}$ The measurement problem consists in explaining why, being the post-experimental state $\left|\psi_{1}\right\rangle$ a superposition of the $\left|a_{i}\right\rangle \otimes\left|p_{i}\right\rangle$, the pointer $\hat{P}$ acquires a definite value, say, its eigenvalue $p_{k}$ corresponding to its eigenstate $\left|p_{k}\right\rangle$.

This formulation of the measurement problem rests on three implicit assumptions: that the state of a system specifies the properties of the system, that the state evolution is linear, and that the measuring apparatus' pointer acquires a single definite value. On this basis, Tim Maudlin (1995) presents what he calls "the problem of outcomes" in the following terms:

"1.A The wave-function of a system is complete, i.e. the wave-function specifies (directly or indirectly) all of the physical properties of a system.

1.B The wave-function always evolves in accord with a linear dynamical equation (e.g. the Schrödinger equation).

1.C Measurements of, e.g., the spin of an electron always (or at least usually) have determinate outcomes, i.e., at the end of the measurement the measuring 
device is either in a state which indicates spin up (and not down) or spin down (and not up)." (Maudlin 1995: 7).

In that same paper, Maudlin mentions other two problems involved in quantum measurement: "the problem of statistics", related to the fact that initial states sometimes lead to different outcomes, whose probabilities are given by the Born rule, and "the problem of effect", concerning the correlations between the outcomes of successive measurements. Although relevant, these two issues will not be considered here because they do not directly concern the present argumentation.

Nevertheless, a further aspect of quantum measurement needs to be taken into account when the phenomenon of decoherence enters the stage. One of the first papers in which Wojciech H. Zurek introduces his notion of environment-induced decoherence is entitled "Pointer basis of quantum apparatus: Into what mixture does the wave packet collapse?" (Zurek 1981). This question reappears in subsequent papers:

"In the real world, even when we do not know the outcome, we do know what the alternatives are, and we can safely act as if only one of them has already occurred. [...] But how can an observer who has not yet consulted the detector express his ignorance about the outcome without giving up his certainty about the "menu" of the possibilities?" (Zurek 1991: 38).

On the basis of Zurek's remarks, in his detailed study of the conceptual meaning of decoherence Maximilian Schlosshauer (2004: 1270) distinguishes two problems regarding quantum measurement. The first one is the problem of definite outcomes: why do measurements have outcomes at all?, why do we perceive a definite value of the pointer and not a superposition of its values? This is the traditional problem as presented in the von Neumann model of measurement and corresponds to Maudlin's problem of outcomes. But the second problem is as relevant as the first one, and is logically previous. The problem of the preferred basis derives from the fact that the expansion of the post-experimental state is in general not unique and, therefore, neither the measured observable nor the apparatus' pointer are uniquely defined. In other words, in eq. (2) the state $\left|\psi_{1}\right\rangle$ can be expressed as a superposition of states belonging to many -in principle infinite- different bases of the Hilbert space $\mathcal{H}_{S} \otimes \mathcal{H}_{M}$ : why the measurement outcome corresponds to the basis defined by measuring apparatus' pointer? Schlosshauer agrees with many other authors in that decoherence does not solve the problem of definite outcomes (see, e.g., Healey 1995, Bub 1997, Pearle 1997, Joos 2000, Adler 2003, Zeh 2003, Bacciagaluppi 2020), but plays a central role in the solution of the problem of the preferred basis: "the preferred basis is not chosen in an ad hoc manner simply to make our measurement records determinate or to match our 
experience of which physical quantities are usually perceived as determinate [...]. Instead the selection is motivated on physical, observer-free grounds" (Schlosshauer 2004: 1280; some authors even question the role of decoherence in the selection of the preferred basis, see Castagnino and Fortin 2011, 2012).

In their paper, Franklin and Seifert explicitly identify the problem of quantum measurement with Maudlin's problem of outcomes. Nevertheless, as explained in the following sections, the problem of the preferred basis must also be considered when the account of molecular structure is addressed from the viewpoint of quantum mechanics.

\section{Enantiomers and Hund's paradox}

As it is well known, enantiomers, also known as optical isomers, are chiral molecules: each member of a pair of enantiomers is not superimposable on its mirror image. The difficulty to explain optical isomerism in quantum terms was already pointed out by Friedrich Hund (1927): chiral states are not eigenstates of the Hamiltonian of the molecule since it is invariant under spatial reflection; so, why do certain chiral molecules display an optical activity that is stable in time, associated to a well-defined chiral state?

Let us consider Hund's paradox in formal terms. The total or "resultant" Hamiltonian $\hat{H}_{R}$ (also called "Coulomb Hamiltonian", see Sutcliffe and Woolley 2012b, 2014) of any molecule takes into account all the interactions among nuclei, among electrons and among electrons and nuclei. Such interactions endow the Hamiltonian with certain symmetries, which are inherited by its eigenstates (see Woolley 1976). Since the Coulombic interaction only depends on the distance between the interacting particles, the Hamiltonian $\hat{H}_{R}$ is symmetric under spatial reflection; therefore, it commutes with the parity operator $\hat{P}$ : $\left[\hat{P}, \hat{H}_{R}\right]=0$. As a consequence, the eigenstates $\left|\Psi_{n}\right\rangle$ of $\hat{H}_{R}$ have parity symmetry: $\hat{P}\left|\Psi_{n}\right\rangle= \pm\left|\Psi_{n}\right\rangle$. In particular, the ground state $\left|\Psi_{0}\right\rangle$ is parity symmetric:

$$
\hat{P}\left|\Psi_{0}\right\rangle=\left|\Psi_{0}\right\rangle \text {. }
$$

Moreover, this feature is preserved during the time evolution of the system because the parity operator commutes with the Hamiltonian $\hat{H}_{R}$ and, as a consequence, is a constant of motion. However, experimentally isomers are not found in states with parity symmetry. In fact, if the two chiral states are represented by the quantum states $|L\rangle$ (left-handed) and $|R\rangle$ (righthanded), the structure of each isomer is the mirror image of the structure of the other:

$$
\hat{P}|L\rangle=|R\rangle \quad \hat{P}|R\rangle=|L\rangle .
$$


Therefore, the states $|L\rangle$ and $|R\rangle$ cannot be eigenstates of the parity-symmetric Hamiltonian $\hat{H}_{R}$. In fact, the ground state should be a superposition of the chiral states; for example,

$$
\left|\Psi_{0}\right\rangle=\frac{1}{\sqrt{2}}(|L\rangle+|R\rangle)
$$

As Franklin and Seifert correctly point out, it is easy to conceive Hund's paradox as a case of the quantum measurement problem: here the issue is to account for the transition from the superposition to one of the chiral states, $|L\rangle$ or $|R\rangle$ (see Fortin et al. 2016, 2018). Therefore, once the quantum measurement problem is solved, Hund's paradox is solved too. Nevertheless, it is crucial not to extrapolate this conclusion to the problem of molecular structure, which consists in explaining the geometrical shape of the molecule in quantum terms. Solving the problem of molecular structure requires that the quantum state -approximately-describes, at least, the positions of all the nuclei in the molecule. But this description is not given by the states $|L\rangle$ and $|R\rangle$. Let us consider this point carefully.

As explained in the previous section, the quantum measurement problem consists in explaining why the pointer $\hat{P}$ acquires a definite value even if the post-experimental state $\left|\psi_{1}\right\rangle$ is a superposition (see eq. (2)). Let us suppose that, via some interpretation of quantum mechanics, one can solve the measurement problem by explaining that the state of the composite system $S+M$ becomes a particular component of the superposition, say, $\left|a_{k}\right\rangle \otimes\left|p_{k}\right\rangle$ and, as a cosequence, the pointer observable $\hat{P}$ acquires its definite eigenvalue $p_{k}$. However, this does not mean that the state $\left|p_{k}\right\rangle$ defines the values of all the microscopic observables of the measuring apparatus. In fact, in practice the apparatus is a macroscopic system, with a huge number of quantum degrees of freedom represented in his Hilbert space $\mathcal{H}_{M}$. So the pointer $\hat{P}$ cannot define a basis of $\mathcal{H}_{M}: \hat{P}$ cannot have a so huge number of eigenvalues because the experimental physicists have to be able to discriminate among them. This means that, in general, the pointer $\hat{P}$ is a "collective observable" (see Omnés 1994, 1999), that is, a highly degenerate observable whose non-unidimensional eigenprojectors introduce a sort of "coarse-graining" on $\mathcal{H}_{M}$. As a consequence, the definite value of $\hat{P}$ does not imply the definite-valuedness of all the microscopic observables of the apparatus.

A situation analogous to that described above in the case of the pointer of the apparatus is that occurring in the case of the observable corresponding to chirality in the molecule. On the one hand, the chiral molecule has many degrees of freedom represented in its resultant Hilbert space $\mathcal{H}_{m}$, in particular, the quantum degrees of freedom related to the observables $\hat{R}_{\alpha}$ corresponding to the positions of the nuclei. On the other hand, in this case the eigenstates of the apparatus' pointer $\hat{P}$ are correlated with the eigenstates $|L\rangle$ and $|R\rangle$ of the "chirality" 
observable $\hat{C}$, whose eigenvalues $r$ and $l$ represent the properties of right-handedness and left-handedness respectively (see Fortin et al. 2018). With its two eigenvalues, $\hat{C}$ is also a highly degenerate "collective observable" in $\mathcal{H}_{m}$, whose definite value -explained by solving the quantum measurement problem- still says nothing about the definite -or almost definitevalues of the nuclei's positions $\hat{R}_{\alpha}$. Broadly speaking, there are many different ways to being a chiral molecule: many different geometrical configurations of the nuclei can realize, say, a left-handed molecule. This means that solving the measurement problem involved in Hund's paradox amounts to explaining the chiral nature of the molecule, but is still far from solving the problem of the molecular structure as a whole.

Summing up, it is clear that Hund's paradox is closely related with the problem of molecular structure. Moreover, we agree with Franklin and Seifert about understanding Hund's paradox as a case of the quantum measurement problem: solving the latter one solves the first one. But solving Hund's paradox does not offer yet a full answer to the problem of molecular structure.

\section{Isomers and resultant Hamiltonian}

In the discussions about the problem of molecular structure, it is usual to distinguish between the complete and the electronic Hamiltonians or, following Robin Hendry terminology, between the resultant and the configurational Hamiltonians of the molecule.

As said in the previous section, the resultant Hamiltonian expresses the Coulombic interactions between all the nuclei and electrons composing the molecule, conceived as quantum entities. In formal terms, it can be written as

$$
\hat{H}_{R}=\hat{T}_{N}\left(\hat{P}_{\alpha}\right)+\hat{V}_{N N}\left(\hat{R}_{\alpha}\right)+\hat{T}_{e}\left(\hat{p}_{i}\right)+\hat{V}_{e e}\left(\hat{r}_{i}\right)+\hat{V}_{e N}\left(\hat{r}_{i}, \hat{R}_{\alpha}\right),
$$

where $\hat{T}_{N}$ is the nuclear kinetic energy (function of the nuclear momenta $\hat{P}_{\alpha}$ ), $\hat{V}_{N N}$ is the potential due to the interactions between the nuclei (function of the nuclear positions $\hat{R}_{\alpha}$ ), $\hat{T}_{e}$ is the electronic kinetic energy (function of the electronic momenta $\hat{p}_{i}$ ), $\hat{V}_{e e}$ is the potential due to the interactions between the electrons (function of the electronic positions $\hat{r}_{i}$ ), and $\hat{V}_{e N}$ is the potential due to the interactions between the electrons and the nuclei (function of the $\hat{r}_{i}$ and the $\hat{R}_{\alpha}$ ). If the nuclei are conceived as fixed at definite position, they lose their quantum character: the now classical nuclear kinetic energy $T_{N}$ is zero, and the nuclear positions are no longer represented by quantum operators $\hat{R}_{\alpha}$ but are given by classical parameters $\boldsymbol{R}_{\alpha}$. In turn, the classical nuclear kinetic energy $T_{n}\left(P_{\alpha}\right)$, now function of the classical nuclear momenta $P_{\alpha}$, is zero, and the classical nuclear potential $V_{n n}\left(\boldsymbol{R}_{\alpha}\right)$ is just a constant that shifts 
the eigenvalues of the new Hamiltonian only by some constant amount and, therefore, can be neglected. As a result, the electronic Hamiltonian turns out to be

$$
\hat{H}_{e}=\hat{T}_{e}\left(\hat{p}_{i}\right)+\hat{V}_{e e}\left(\hat{r}_{i}\right)+\hat{V}_{e N}\left(\hat{r}_{i}, \boldsymbol{R}_{\alpha}\right)=\hat{H}_{e}\left(\hat{r}_{i}, \boldsymbol{R}_{\alpha}\right) .
$$

With this Hamiltonian the time-independent Schrödinger equation results

$$
\hat{H}_{e}\left(\hat{r}_{i}, \boldsymbol{R}_{\alpha}\right) \psi_{n}\left(\hat{r}_{i}, \boldsymbol{R}_{\alpha}\right)=E_{n}\left(\boldsymbol{R}_{\alpha}\right) \psi_{n}\left(\hat{r}_{i}, \boldsymbol{R}_{\alpha}\right),
$$

where the $\psi_{n}\left(\hat{r}_{i}, \boldsymbol{R}_{\alpha}\right)$ are the electronic eigenstates and the $E_{n}\left(\boldsymbol{R}_{\alpha}\right)$ are the electronic eigenvalues for fixed $\boldsymbol{R}_{\alpha}$, with $n=0$ corresponding to the electronic ground state. If this calculation is repeated by varying $\boldsymbol{R}_{\alpha}$, an effective potential $E_{n}\left(R_{\alpha}\right)$ is obtained, where now the $R_{\alpha}$ are no longer parameters but variables; on $E_{n}\left(R_{\alpha}\right)$ electrons supposedly move. So, for the nuclear degrees of freedom, a nuclear Hamiltonian $\hat{H}_{N n}$ for the $n^{\text {th }}$ effective potential can be constructed by turning back the nuclear positions into operators:

$$
\hat{H}_{N n}\left(\hat{R}_{\alpha}\right)=\hat{T}_{N}\left(\hat{P}_{\alpha}\right)+\hat{E}_{n}\left(\hat{R}_{\alpha}\right) .
$$

Now, an effective or configurational Hamiltonian of the molecule can be defined as

$$
\hat{H}_{C}\left(\hat{r}_{i}, \hat{R}_{\alpha}\right)=\hat{H}_{e}\left(\hat{r}_{i}, \boldsymbol{R}_{\alpha}\right)+\hat{H}_{N n}\left(\hat{R}_{\alpha}\right),
$$

which is no longer Coulombic and, as a consequence, does not have the same symmetries as the resultant Hamiltonian: "the conventional product of the electronic wavefunctions (from the clamped-nuclei Hamiltonian) and associated nuclear wavefunctions lack the symmetry properties of Coulomb Hamiltonian eigenfunctions and this difference has not been explained." (Sutcliffe and Woolley 2021: forthcoming).

The use of the BOA is pervasive in quantum chemistry, and this fact must not be questioned: its justification relies in its own success. Here the question is how the entire strategy is viewed from the perspective of quantum mechanics.

The first point to notice is that, from the viewpoint of quantum mechanics, each quantum system has its own Hamiltonian: changing the Hamiltonian amounts to changing the identity of the quantum system itself. So, if one decides to replace a set of the variables by fixed numbers and associate these numbers with infinite mass and the same charge as the variables replaced had, one gets a new problem (Sutcliffe and Woolley, personal communication). As a consequence, strictly peaking, the only Hamiltonian of the quantum molecule is the resultant one. The configurational Hamiltonian corresponds to a different quantum system: a system of quantum electrons interacting between them and moving in an effective non-Coulombic potential. Therefore, the configurational Hamiltonian not merely "impose certain restrictions on the behaviour of these particles [the entities that comprise the 
molecule]" (Franklin and Seifert 2020: 8-9): the interactions between the entities that comprise the molecule change their very nature and, as a consequence, the quantum system described by the configurational Hamiltonian is not the same as that described by the resultant Hamiltonian. Franklin and Seifert are right when they say that "[a]fter applying the BO approximation one can, in principle, formulate quantum descriptions for all the possible fixed positions of the nuclei." (2020: 11). However, they add: "Each assignment of positions to nuclei corresponds to different quantum states of the system" (2020: 11). The question is: what system? In fact, the system corresponding to the configurational Hamiltonian is no longer the quantum molecule -the system composed of quantum nuclei and quantum electrons with Coulombic interactions-, but a system constituted by quantum electrons and semi-classical nuclei in an effective Born-Oppenheimer potential.

The second point is how to understand the "clamped nuclei" strategy, which is the core of the Born-Oppenheimer approximation, from the viewpoint of quantum mechanics. The problem of its justification in quantum terms will be not treated here because it has been discussed in detail elsewhere (Lombardi and Castagnino 2010). Nevertheless, it cannot be ignored that assuming the nuclei to be placed at rest at definite positions contradicts the Heisenberg principle. Somebody might retort that the nuclear kinetic energy $\hat{T}_{n}\left(\hat{P}_{\alpha}\right)$ tends to zero because the nuclear masses tend to infinite (when compared with electronic masses) and not because the nuclear momenta tend to zero; then, it is not necessary to suppose that the nuclei are at rest. However, from a strict quantum perspective, if the positions $\hat{R}_{\alpha}$ of the nuclei have precise values, the values of the momenta $\hat{P}_{\alpha}$ are completely indefinite. In other words, assuming that the nuclei are placed at definite positions and that the values of their momenta are bounded to meaningfully make that $\hat{T}_{n}\left(\hat{P}_{\alpha}\right)$ tends to zero also contradicts the Heisenberg principle, which establishes a finite bound to the corresponding uncertainties. Therefore, the BOA not only "simply assume the facts about molecular structure that ought to be explained" (Hendry 2010: 186). It also introduces a counterfactual approximation (see Bruer 1982, Rohrlich 1989), which, by contrast with a factual approximation, contradicts a postulate of the theory. A counterfactual approximation is legitimate only if it can be replaced by a factual approximation; for example, in special relativity the counterfactual limit $c \rightarrow \infty$ is legitimate because it can be replaced by a factual limit $v / c \rightarrow 0$, that is, an approximation for velocities $v$ much lower than the speed of light $c$. But the BOA, which leads to supplant the quantum nuclear positions $\hat{R}_{\alpha}$ by the classical nuclear positions $R_{\alpha}$, cannot be replaced by any factual approximation in the context of quantum mechanics. Hasok Chang clearly makes the point: 
"In this «clamping-down» approximation, the atomic nuclei are treated essentially as classical particles; [...] this picture is non-quantum in a very fundamental way as the simultaneous assignment of fixed positions and fixed momenta (namely, zero) to them violates the Heisenberg uncertainty principle. But without such classical scene-setting, the quantum calculations are quite impossible.

The difficulty here is not only about the practicalities of calculation, and the clamping-down of nuclei is not merely an approximation. Aside from assuming that the nuclei are fixed, it is necessary to know where exactly the nuclei in question should be placed. Otherwise it is not possible to specify the potential function, which needs to be inserted into the Schrödinger equation, whose solution determines the wavefunction of the electrons in the molecule." (Chang 2015: 198).

(for a detailed discussion of the qualitative difference between classical identifiable nuclei and nuclei as quantum entities, see Sutcliffe and Woolley 2012b, 2014).

The appeal to the configurational Hamiltonian, obtained through the BOA, is due to the fact that the resultant Hamiltonian is unable to account for the asymmetry of certain molecules. In particular, it cannot distinguish among different isomers: since they are quantum systems with the same number and types of elements, isomers are described by the same resultant Hamiltonian. By contrast, "one can understand the role of the configurational Hamiltonian as picking out that structure which agrees with the result of a particular measurement." (Franklin and Seifert 2020: 20). According to the authors, "[e]ven though a resultant Hamiltonian does not determine the molecular structure that is identified empirically (that is, by measurement), it nevertheless, at least in principle, determines all possible structures of the examined molecule" (2020: 20), and the reason is that "the system is in a superposition of such [molecular] configurations." (2020: 21). Does it mean that the ground state of the resultant Hamiltonian is the superposition of the ground states of the configurational Hamiltonians corresponding to the different isomeric configurations? Of course, not. As explained above, the resultant Hamiltonian $\hat{H}_{R}$ is the quantum Hamiltonian of the molecule, whereas the configurational Hamiltonians describe different systems, resulting from the application of the semiclassical BOA. So, it is difficult to see how the ground state of a system turns out to be the superposition of the ground states of different systems.

With all that said, let us forget the configurational Hamiltonian and focus on the resultant Hamiltonian. Let us consider the idea that "that the ground state wavefunction of the resultant Hamiltonian corresponds to a superposition of all the different isomers." (Franklin and Seifert 2020: 12) and express it in formal terms. The resultant Hamiltonian defines its eigenstates $\left|\Psi_{n}\right\rangle$ and eigenvalues $E_{n}, \hat{H}_{R}\left|\Psi_{n}\right\rangle=E_{n}\left|\Psi_{n}\right\rangle$, with $\left|\Psi_{n}\right\rangle \in \mathcal{H}_{m}=\mathcal{H}_{e} \otimes \mathcal{H}_{N}$, and the ground state is the resultant Hamiltonian's eigenstate $\left|\Psi_{0}\right\rangle$ corresponding to the minimum 
energy value $E_{0}$. Now, we are said that we can conceive $\left|\Psi_{0}\right\rangle$ as a superposition of the states $\left|\varphi_{i}\right\rangle$ corresponding to the different isomers,

$$
\left|\Psi_{0}\right\rangle=\sum_{i} c_{i}\left|\varphi_{i}\right\rangle
$$

According to Franklin and Seifert, the experimental identification of a particular isomer is a quantum measurement process that "selects" one of the components from the whole superposition; therefore, solving the quantum measurement problem implies solving the problem of the molecular structure in the case of isomers. The idea is certainly very ingenious, but it needs to be analyzed with care.

A point to be noticed here is that the authors conceive the measurement problem as the problem of -definite- outcomes. However, the problem of the preferred basis must also be taken into account. In fact, the Hilbert space $\mathcal{H}_{m}$ has infinite basis, and the ground state $\left|\Psi_{0}\right\rangle$ can be expressed as a superposition of the members of any of them: why the measurement outcome corresponds to the basis constituted by the $\left|\varphi_{i}\right\rangle$ and not to any other basis? The answer to this question can be given by appealing to the interaction of the molecule with the environment; this point will be considered in the next section. Here it is sufficient to stress that conceiving the problem of molecular structure in the case of isomers as a case of the quantum measurement problem also requires solving the preferred basis problem, and not only the problem of definite outcomes.

Granted that the previous considerations have been taken into account, a further issue comes to the fore, which threatens Franklin and Seifert's claims. The resultant Hamiltonian $\hat{H}_{R}$ of the molecule has certain symmetries regarding the nuclear degrees of freedom that are necessarily inherited by its eigenstates, in particular, by the ground state $\left|\Psi_{0}\right\rangle$. On the other hand, in general the isomeric structures, given the positions of their nuclei, break at least some of those symmetries; therefore, the $\left|\varphi_{i}\right\rangle$ corresponding to the different isomeric structures do not have all the symmetries of $\hat{H}_{R}$. It is precisely for this reason that some authors consider that there is an in-principle obstacle to account for molecular structure exclusively in quantum terms (see e.g. Woolley 1978, 1998, Hendry 2010). Pierre Claverie and Simon Diner (1980), in a paper repeatedly referred to by Franklin and Seifert, clearly summarize the problem as understood by Guy Woolley:

"It is not possible to associate a definite molecular structure with a molecule described by an exact (time independent) eigenstate of the complete molecular Hamiltonian. By complete, we mean that all kinetic and potential terms are kept for all particles, electrons and nuclei as well, and that consequently the corresponding eigenfunctions are functions of all electronic and nuclear coordinates. Basically, Woolley argues that the symmetry inherent in this 
complete Hamiltonian as concerns the nuclei (rotational symmetry, reflection symmetry, permutational symmetry for identical nuclei), which is necessarily reflected in the eigenfunctions (notably the ground state one), precludes the existence of a «molecular structure»." (Claverie and Diner 1980: 58; italics in the original).

As already explained, the solution offered by Franklin and Seifert relies on conceiving the symmetric ground state $\left|\Psi_{0}\right\rangle$ as a superposition of the asymmetric $\left|\varphi_{i}\right\rangle$ corresponding to the different isomeric structures. But it must be stressed that, in order to recovering the symmetry of $\left|\Psi_{0}\right\rangle$ from the superposition of the asymmetric $\left|\varphi_{i}\right\rangle$, it is necessary to include all the mathematically possible isomeric structures in the superposition, that is, all the mathematically possible nuclear configurations, with the same probability. In other words, in eq. (11), the $\left|\varphi_{i}\right\rangle$ must represent all the possible relative positions of the nuclei -besides all the possible electronic configurations- and all the coefficients $c_{i}$ must be equal: it is only by superposing all possible asymmetries with equal weight that a symmetry can be reconstructed. This theoretical fact should be empirically reflected by obtaining -at least, approximately- all the possible nuclear configurations with the same frequency in effective experiments. The problem is that this is not the case: given the number and the types of the elements composing a molecule, in general only few nuclear configurations are experimentally measured, that is, only few isomers are considered effectively "real". Of course, there are many chemical reasons for the existence of certain isomers and not of others. But, from the viewpoint of quantum mechanics the question is: Why certain isomeric structures are obtained in laboratory, and not all those necessary to reconstruct the symmetry of the ground state? This question is at the core of the problem of molecular structure, and it is not answered by solving the quantum measurement problem precisely because its answer is necessary to formulate the problem of molecular structure as a case of the quantum measurement problem.

Finally, let us suppose that the above problem could be solved and one of the asymmetric $\left|\varphi_{i}\right\rangle$, representing a "real" isomer, were obtained; this would be a Pyrrhic victory. In fact, in Franklin and Seifert's argument, the ground state $\left|\Psi_{0}\right\rangle$ is a non-trivial superposition of the $\left|\varphi_{i}\right\rangle$; as a consequence, the $\left|\varphi_{i}\right\rangle$ are not eigenstates of the resultant Hamiltonian $\hat{H}_{R}$ of the molecule. This means that, even if immediately after measurement the molecule is, say, in the particular state $\left|\varphi_{k}\right\rangle$, at subsequent times it will evolve to different states according to the -time-dependent- Schrödinger equation. By contrast, in chemistry it is assumed that structure is a stable property of the molecule, at least stable enough as to be described in terms of the geometrical arrangement of the nuclei which can be measured with great precision. As Claverie and Diner explicitly stress: 
"How can we explain that, in the course of time, these [initial localized] states retain their localized character? [...] even if we are prepared to concede that the "localized" state, different from the molecular eigenstates, has been created by this interaction, [...] the molecular Hamiltonian alone is unable to maintain this "localized" character in the course of time." (Claverie and Diner 1980: 68).

The stability of molecules that are not in the ground state of the resultant Hamiltonian cannot be easily explained exclusively in quantum terms: this is a central aspect of the problem of molecular structure that is not related with the quantum measurement problem, at least in Maudlin's version.

Franklin and Seifert "argue that the apparent in-principle inability of resultant Hamiltonians to determine observed structure is a special case of the measurement problem, and that it is resolved by putative resolutions of the measurement problem." (2020: 9). The above discussion about the case of isomerism shows that this is a hasty conclusion: there are aspects of the problem of molecular structure that are not captured by the problem of quantum measurement as the authors present it.

\section{Symmetry breaking}

Franklin and Seifert say that, when compared with the two previous problems, the issue of symmetry breaking "is less often considered as a «problem of molecular structure»." (2020: 13). However, as they themselves notice, symmetry breaking was seriously discussed by the authors who most dealt with the problem of molecular structure. Following Guy Woolley and Brian Sutcliffe (1977), Hendry (2010) clearly characterizes what he calls "the symmetry problem": since the interactions embodied in the resultant Hamiltonian of the molecule are Coulombic, the solutions of the Schrödinger equation (the eigenstates of the resultant Hamiltonian) are rotationally symmetric; however, the rotational asymmetry of polyatomic molecules is essential in the explanation of their chemical behavior (see also Sutcliffe and Woolley 2012a). As Hendry stresses, in the quantum domain no directional property can be assigned to an isolated molecule in an eigenstate of the resultant Hamiltonian. For example, the hydrogen chloride molecule has an asymmetrical charge distribution that explains its acidic behavior and its boiling point; however, according to quantum mechanics, the expectation value of the electric dipole moment of the molecule in an arbitrary eigenstate of the resultant Hamiltonian is always zero: "the acidic behaviour of the hydrogen chloride molecule is conferred by its asymmetry", but "the asymmetry is not conferred by the molecule's physical basis according to physical laws" (Hendry 2010: 186, see also Hendry 
1998; for a precise account of the experimental distinction between polar and non-polar molecules, see Woolley 1976).

Instead of considering the case of hydrogen chloride, Franklin and Seifert appeal to the example of the ammonia molecule, whose tetrahedral structure -with the negative nitrogen in one of the vertices and the positive hydrogens in the other three- accounts for its electric dipole moment. They rely on the explanation given by Philip Warren Anderson: since no stationary state of the molecule can have a nonzero value of the electric dipole moment, the molecule "oscillates" between two asymmetric states: "By means of quantum mechanical tunneling, the nitrogen can leak through the triangle of hydrogens to the other side [...] A truly stationary state can only be an equal superposition of the unsymmetrical pyramid and its inverse." (Anderson 1972: 394). Anderson's explanation appeals to the so-called nitrogen inversion or umbrella inversion: a fluxional process by which the nitrogen "moves" through the plane formed by the hydrogens and the molecule "turns inside out" (nitrogen inversion is not exclusive of ammonia, but is characteristic of other kinds of molecules). However, it is quite clear that this is a semi-classical explanation, since already assumes the geometrical structure of the molecule: at each time the positions of the nuclei are definite and, as a consequence, the molecule has a precise shape. Therefore, it cannot be used in the context of the problem of accounting for molecular structure in quantum terms.

Franklin and Seifert notice that "Anderson's account of symmetry breaking is hardly mentioned in the literature on the problems considered in sections 2.1-2.2 above [those discussed in Sections 3 and 4 of the present paper], though a notable exception is Claverie and Diner (1980)." (2020: 15). But in that paper Claverie and Diner explicitly stress that this kind of explanation is not quantum; let us consider what they say about Anderson's account of symmetry breaking:

"it is tempting to consider that the nitrogen nucleus lies, at any given time, on a well-defined side of the plane of the three protons, and that it jumps from one side to the other at more or less regular intervals of time. Of course, this «naive» view is at variance with strict quantum theory, but it is nevertheless very commonly used, either for teaching purposes or in popularization papers (see e.g. Anderson 1972)." (Claverie and Diner 1980: 74).

We want to stress that we do not agree with the qualification "naïve": the fact that an explanation is non-quantum does not make it naïve. On the contrary, the nitrogen-inversion model gives a chemical significant account of a phenomenon that has been accurately measured. What must be stressed is that the nitrogen-inversion model, with the oscillation between the two structures, is a semi-classical explanation: a system in a quantum 
superposition state does not oscillate between the two components of the superposition. More precisely:

"On the classical theory we picture the nitrogen atom flipping back and forth at a characteristic frequency of about 24,000 million vibrations per second, or 24,000 megacycles per second. At any given instant the nitrogen atom is on one side of the hydrogens or on the other. From the quantum point of view the nitrogen has at a given time a certain probability of being on either side" (Gordon 1958: 42).

If the issue at stake is the problem of molecular structure, the question is how to explain the nonzero value of the electric dipole moment exclusively in quantum terms and, so, semiclassical descriptions must be excluded.

When talking about symmetry breaking, it is necessary to be clear about which symmetry is broken. Following with the idea of characterizing the problem of molecular structure as a case of the quantum measurement problem, Franklin and Seifert need to conceive the symmetric ground state of the molecule as a superposition of asymmetric states: "the wavefunction representing a superposition of the pyramid and its inversion is the best way to describe ammonia in the quantum formalism." (2020: 22). If the symmetric ground state of the ammonia molecule is expressed as a superposition of two asymmetric states representing the pyramid and its inversion, the symmetry under consideration is parity symmetry, as Anderson immediately specifies regarding his previous explanation: "The symmetry involved in the case of ammonia is parity, the equivalence of left- and right-handed ways of looking at things" (1972: 394). So, this case is similar to that of optical isomerism: here the role of the measuring pointer is played by an observable $\hat{D}_{z}$, "direction of the electric dipole moment in $z "$, whose eigenstates are $\left|\mu_{+}(z)\right\rangle$ and $\left|\mu_{-}(z)\right\rangle$ and whose eigenvalues $\mu_{+}(z)$ and $\mu_{-}(z)$ represent the moment pointing "up" and "down" in direction $z$, respectively. Again, asymmetric states $\left|\mu_{+}(z)\right\rangle$ and $\left|\mu_{-}(z)\right\rangle$ cannot be eigenstates of the parity-symmetric resultant Hamiltonian $\hat{H}_{R}$ of the molecule: the ground state $\left|\Psi_{0}\right\rangle$ of $\hat{H}_{R}$ can be expressed as a superposition of those two asymmetric states (see the analogous eq. (5)):

$$
\left|\Psi_{0}\right\rangle=\frac{1}{\sqrt{2}}\left(\left|\mu_{+}(z)\right\rangle+\left|\mu_{-}(z)\right\rangle\right) \text {. }
$$

This can also be conceived as a case of the quantum measurement problem: here the issue is to account for the transition from the superposition to one of the asymmetric states, $\left|\mu_{+}(z)\right\rangle$ and $\left|\mu_{-}(z)\right\rangle$, with definite value of $\hat{D}_{z}$, the direction of the electric dipole moment in $z$. But, as in the case of optical isomerism, in the Hilbert space $\mathcal{H}_{m}$ corresponding to the molecule $\hat{D}_{z}$ is also a highly degenerate "collective observable", whose definite value -explained by solving the quantum measurement problem- have no relevance regarding the values of the 
nuclei's positions $\hat{R}_{\alpha}$. Therefore, also in this case, solving the measurement problem explains the breaking of parity symmetry, but does not offer a complete solution of the problem of the molecular structure

We stressed the relevance of specifying which symmetry one is talking about because Franklin and Seifert confuse parity symmetry, that treated by Anderson, with rotational symmetry:

"The issue explored by Anderson is that quantum mechanics predicts that molecules should have certain symmetry properties, which the molecules are observed to violate. In the particular example raised, Anderson asks how it is that ammonia exhibits an electric dipole, with a preferred direction, when the quantum mechanical description is rotationally symmetric -a description with no preferred direction and no dipole moment." (Franklin and Seifert 2020: 14).

In fact, the rotational symmetry of the resultant Hamiltonian $\hat{H}_{R}$ is usually considered when the problem of molecular structure is discussed, but conceiving rotational symmetry breaking as a case of quantum measurement problem cannot be explained as in the previous paragraph: the superposition is not given by eq. (12). In order to recover the rotational symmetry that the ground state $\left|\Psi_{0}\right\rangle$ inherits from $\hat{H}_{R}$, the superposition must include the eigenstates of all the observables $\hat{D}_{z}$ defined for the infinite spatial directions, so that $\left|\Psi_{0}\right\rangle$ is not expressed as eq. (12) but as

$$
\left|\Psi_{0}\right\rangle=\int_{0}^{\pi} \int_{0}^{\pi / 2} c(\theta, \phi)\left(\left|\mu_{+}(\theta, \phi)\right\rangle+\left|\mu_{-}(\theta, \phi)\right\rangle\right) d \theta d \phi
$$

where $c(\theta, \phi)$ is a constant with the value $\pi^{-1}$. Analogously to the case of isomers discussed in the previous section, the symmetry is reconstructed by superposing all the possible asymmetries with equal weight. But by contrast to the isomers case, here the superposition is experimentally justified: if the direction of the electric dipole moment is measured in all spatial directions (for instance, by measurements involving an electric field in such directions), the dipole moment will be found oriented in all the directions with the same frequency. Therefore, also in this case the experimental identification of the direction of the electric dipole moment is a quantum measurement process that "selects" one of the components from the whole superposition, now represented by eq. (13). Does it mean that, at least in this case, the solution of the quantum measurement problem solves the problem of molecular structure? The answer is once again negative: as explained in the previous paragraph, the definite value of the direction of the electric dipole moment operator in a particular spatial direction does not univocally determine the molecular structure: there are many geometrical dispositions of the nuclei that lead to the same direction of the electric dipole moment. In Woolley's words: 
"no amount of computational investigation of the full molecular Schr6dinger equation could lead to, for example, the familiar chemical idea of ammonia as a pyramidal molecule which is inverting, because the two modes of description have nothing in common." (Woolley 1976: 32).

Finally, it's time to consider the idea, repeated in several cases, that the isolated molecule has no structure and that symmetry breaking is the result of the interaction between the molecule and its environment (see, traditionally, Woolley 1978; more recently, Fortin et al. 2016, 2018). However, this claim needs to be taken with care, because its soundness depends on the particular kind of environment considered. For instance, the case of sugar mentioned by Franklin and Seifert is analogous to that of many pharmacological drugs: they are chiral, and generally only one of the members of the pair exhibits biological activity. The reason is that protein molecules are chiral and, as a consequence, have different reactions with the two enantiomers of a chiral sugar or drug molecule. But now the problem is to explain the chiral nature of the biological molecules. In general, given the symmetries of a given molecule $M$, if the environment is also composed of molecules, there are only two alternatives: (i) the environmental molecules are also symmetric, so they cannot break the symmetry of $M$, or (ii) the environmental molecules break the symmetry of $M$ because they are asymmetric, but then the problem is moved one step back because now the asymmetry of the environmental molecules cries for an explanation. As Hendry clearly states:

"The particular form of the symmetry-breaking addition must be justified however, and it is quite mysterious how that could work if all one has in the environment are more molecules described by Coulombic Hamiltonians. The Coulomb Schrödinger equation for an n-molecule ensemble of hydrogen chloride molecules has precisely the same symmetry properties as a Coulomb Schrödinger equation for a 1-molecule system. If the particular form of the symmetry-breaking addition is not justified, then it is just ad hoc: a deus ex machina." (Hendry 2010: 186).

Some authors put their faith in decoherence to solve the quantum measurement problem, specifically, the problem of definite outcomes. If this were the case, from Franklin and Seifert's perspective decoherence would also solve the molecular problem since they conceive it as a particular case of the problem of outcomes. But, as stressed in Section 2, there is a wide agreement among physicists and philosophers of physics in that, although decoherence plays a central role in the problem of the preferred basis, it does not offer a solution to the problem of definite outcomes (the traditional measurement problem). This issue has been discussed in detail elsewhere (Fortin et al. 2016) in the case of enantiomers, but the conclusions can be extended to other cases of symmetry breaking. Nevertheless, even if one accepted that decoherence not only selects the preferred basis but also explains the measurements' definite outcomes, she would not be in a better position regarding the problem of molecular structure 
for the same reasons as those explained in this and the previous sections: solving the quantum measurement problem is not sufficient to solve the problem of molecular structure.

The assumption that the interaction with the environment is responsible for the breaking of the molecule's symmetries cannot be a mere general idea, but needs to be developed in precise physical terms. The molecule has several different symmetries, and each of them requires a specific environment able to break each particular one. For example, polarized light is an appropriate environment to break the parity symmetry of chiral molecules, and an electric field in a particular direction is adequate to break the rotational symmetry that blocks the existence of an electric dipole moment. However, it is worth emphasizing again that, although in these cases any answer to the quantum measurement problem gives an account to the symmetry breaking, such an account does not solve the problem of molecular structure: it only explains the definite value of some particular observable, but not the geometry of the molecule partially given by the spatial position of its nuclei nor the stability of that geometry.

The idea that quantum measurement is strongly linked with symmetry breaking is not new (see, e.g., Lombardi and Castagnino 2008, Lombardi 2018), even in the case of quantum chemistry (Fortin et al. 2016, 2018). What is new in Franklin and Seifert's paper is the claim that "insofar as the measurement problem is solved, the problems of molecular structure are resolved as well" (Franklin and Seifert 2020, Abstract). But it is precisely this claim that cannot be easily defended.

\section{Conclusions}

An undoubted virtue of the Franklin and Seifert's article is its contribution to the communication between the philosophy of chemistry and the philosophy of physics, which, as the authors point out, have a rather small overlap. Another positive aspect is the fact that it brings to the fore, once again, the relationships among symmetry, quantum measurement, and molecular structure. Nevertheless, the argumentation has weak points when semi-classical notions (nitrogen inversion, configurational Hamiltonian) are appealed to in a context that should be confined to quantum mechanics, and when the particular symmetry to be broken in each case under study is not correctly identified. However, the main limitation of the article is that its main claim, expressed in its title -"The problem of molecular structure just is the measurement problem"- is not correctly justified in any of the three cases treated by the authors. 
- In the cases of symmetry breaking (Section 5) and of enantiomers (a special case of the first one; Section 3), an adequate solution to the quantum measurement problem would explain how the different symmetries of the resultant Hamiltonian can be broken through an appropriate measurement interaction. Still, a particular symmetry breaking only justifies the definite value of the observable involved in that symmetry, but does not explain the geometrical disposition of the nuclei in space in order to account for the molecular structure.

- In the general case of isomers (Section 4), the solution of the problem of molecular structure is even further away than in the previous case: the fact that certain isomeric structures, and not all the possible ones, are empirically observed cannot be explained even if one counts with an adequate solution to the quantum measurement problem.

Franklin and Seifert's argumentation does not stop in their main claim, but takes one more step towards the issue of the relationship between chemistry and physics. In fact, the inability of the resultant Hamiltonian to account for the molecular asymmetries has been traditionally invoked to argue against the reduction of molecular chemistry to quantum mechanics. The authors consider to have shown that, since the problem of molecular structure just is the quantum measurement problem, isolated molecules have no structure. Of course, if the solution of the measurement problem not only resolved the symmetry problem -which we are willing to subscribe-, but also supplied an answer to the whole problem of molecular structure, a strong argument for reductionism would be obtained: "the identification of the problems of molecular structure as special cases of the measurement problem removes significant hurdles facing the reductionist." (Franklin and Seifert 2020: 25). Nevertheless, this is not a case. In fact, although we agree in that the symmetry problem can be successfully confronted in quantum terms by solving the measurement problem, we have shown that serious in-principle difficulties still remain when trying to reduce molecular structure to quantum mechanics.

\section{Acknowledgements}

We are strongly indebted to Brian Sutcliffe and Guy Woolley for devoting their time to discussing the problem of molecular structure with us and for reading this article: their comments are always a source of invaluable teachings. This work was supported by grant PICT-04519 of the Agencia Nacional de Promoción Científica y Tecnológica of Argentina. 


\section{References}

Adler, S. (2003). "Why decoherence has not solved the measurement problem: A response to P. W. Anderson.” Studies in History and Philosophy of Modern Physics, 34: 135-142. Amann, A. (1992). "Must a molecule have a shape?" South African Journal of Chemistry, 45: 29-38.

Anderson, P. W. (1972). "More is different.” Science, 177: 393-396.

Bacciagaluppi, G. (2020). "The role of decoherence in quantum mechanics." In E. N. Zalta (ed.), The Stanford Encyclopedia of Philosophy (Fall 2020 Edition), $<$ https://plato.stanford.edu/archives/fall2020/entries/qm-decoherence/>.

Bader, R. (1990). Atoms in Molecules. A Quantum Theory. Oxford: Oxford University Press.

Bader, R. (2011). "On the non-existence of parallel universes in chemistry." Foundations of Chemistry, 13: 11-37.

Bruer, J. T. (1982). “The classical limit of quantum theory.” Synthese, 50: 167-212.

Bub, J. (1997). Interpreting the Quantum World. Cambridge: Cambridge University Press.

Castagnino, M. and Fortin, S. (2011). "New bases for a general definition for the moving preferred basis." Modern Physics Letters A, 26: 2365-2373.

Castagnino, M. and Fortin, S. (2012). "Non-Hermitian Hamiltonians in decoherence and equilibrium theory." Journal of Physics A: Mathematical and Theoretical, 45: 444009.

Chang, H. (2015). "Reductionism and the relation between chemistry and physics." Pp. 193210 in T. Arabatzis, J. Renn, and A. Simoes (eds.), Relocating the History of Science: Essays in Honor of Kostas Gavroglu. New York: Springer.

Claverie, P. and Diner, S. (1980). "The concept of molecular structure in quantum theory: interpretation problems." Israel Journal of Chemistry, 19: 54-81.

Fortin, S., Lombardi, O., and Martínez González, J. C. (2016). "Isomerism and decoherence." Foundations of Chemistry, 18: 225-240.

Fortin, S., Lombardi, O., and Martínez González, J. C. (2018). "A new application of the modal-Hamiltonian interpretation of quantum mechanics: The problem of optical isomerism." Studies in History and Philosophy of Modern Physics, 62: 123-135.

Franklin, A. and Seifert. V. (2020). "The problem of molecular structure just is the measurement problem." Forthcoming in The British Journal for the Philosophy of 
Science. Page numbers taken from The Problem of Molecular Structure Just Is The Measurement Problem - Research Portal, King's College, London (kcl.ac.uk).

Gordon, J. P. (1958). “The maser.” Scientific American, 199: 42-51.

Healey, R. A. (1995). "Dissipating the quantum measurement problem.” Topoi, 14: 55-65.

Hendry, R. F. (1998). “Models and approximations in quantum chemistry.” In N. Shanks (ed.), Idealization in Contemporary Physics. Amsterdam-Atlanta: Rodopi.

Hendry, R. F. (2004). "The physicists, the chemists, and the pragmatics of explanation." Philosophy of Science, 71: 1048-59.

Hendry, R. F. (2008). "Two conceptions of the chemical bond." Philosophy of Science, 75: 909-920.

Hendry, R. F. (2010). “Ontological reduction and molecular structure.” Studies in History and Philosophy of Modern Physics, 41: 183-191.

Hettema, H. (2012). Reducing Chemistry to Physics. Limits, Models, Consecuences. Groningen: University of Groningen.

Hund. F. (1927). “Zur Deutung der Molekelspektren. III.” Zeitschrift für Physik, 43: 805-826.

Joos, E. (2000). "Elements of environmental decoherence." Pp. 1-17 in P. Blanchard, D. Giulini, E. Joos, C. Kiefer, and I.-O. Stamatescu (eds.), Decoherence: Theoretical, Experimental, and Conceptual Problems, Lecture Notes in Physics, Vol. 538. Heidelberg-Berlin: Springer.

Lombardi, O. (2018). "The Modal-Hamiltonian Interpretation: measurement, invariance and ontology.” Pp. 32-50 in O. Lombardi, S. Fortin, C. López, and F. Holik (eds.), Quantum Worlds. Perspectives on the Ontology of Quantum Mechanics. Cambridge: Cambridge University Press.

Lombardi, O. and Castagnino, M. (2008). "A modal-Hamiltonian interpretation of quantum mechanics." Studies in History and Philosophy of Modern Physics, 39: 380-443.

Lombardi, O. and Castagnino, M. (2010). "Matters are not so clear on the physical side." Foundations of Chemistry, 12: 159-166.

Martínez González, J. C., Fortin, S., and Lombardi, O. (2019). "Why molecular structure cannot be strictly reduced to quantum mechanics." Foundations of Chemistry, 21: 31-45. 
Matta, C. F., Lombardi, O., and Jaimes Arriaga, J. (2020). "Two-step emergence: the quantum theory of atoms in molecules as a bridge between quantum mechanics and molecular chemistry." Foundations of Chemistry, 22: 107-129.

Maudlin, T. (1995). “Three measurement problems.” Topoi, 14: 7-15.

Myrvold, W. (2018). "Philosophical issues in quantum theory." In E. N. Zalta (ed.), The Stanford Encyclopedia of Philosophy (Fall 2018 Edition), $<$ https://plato.stanford.edu/archives/fall2018/entries/qt-issues/>.

Omnés, R. (1994). The Interpretation of Quantum Mechanics. Princeton: Princeton University Press.

Omnés, R. (1999). Understanding Quantum Mechanics. Princeton: Princeton University Press. Pearle, P. (1997). “True collapse and false collapse.” Pp. 51-68 in D. H. Feng and B.-L. Hu (eds.), Quantum Classical Correspondence: Proceedings of the 4th Drexel Symposium on Quantum Nonintegrability, Philadelphia. Cambridge, MA: International Press.

Primas, H. (1983). Chemistry, Quantum Mechanics and Reductionism. Berlin: Springer.

Primas, H. (1998). "Emergence in exact natural sciences." Acta Polytechnica Scandinavica, 91: 83-98.

Rohrlich, F. (1989). "The logic of reduction: The case of gravitation.” Foundations of Physics, 19: $1151-1170$.

Scerri, E. R. (2011). “Editorial 37.” Foundations of Chemistry, 13: 1-7.

Scerri, E. R. (2013). "Philosophy of chemistry: where has it been and where is it going." In J.P. Llored (ed.), The Philosophy of Chemistry: Practices, Methodologies, and Concepts. Newcastle: Cambridge Scholars Publishing.

Schlosshauer, M. (2004). "Decoherence, the measurement problem, and interpretations of quantum mechanics.” Reviews of Modern Physics, 76: 1267-1305.

Sutcliffe, B. T. and Woolley, R. G. (2011). “A comment on Editorial 37.” Foundations of Chemistry, 13: 93-95.

Sutcliffe, B. T. and Woolley, R. G. (2012a). "Atoms and molecules in classical chemistry and quantum mechanics.” In R. F. Hendry and A. Woody (eds), Handbook of Philosophy of Science. Vol. 6, Philosophy of Chemistry. Oxford: Elsevier. 
Sutcliffe, B. T. and Woolley, R. G. (2012b). "On the quantum theory of molecules." The Journal of Chemical Physics, 137: 22A544.

Sutcliffe, B. T. and Woolley, R. G. (2014). "Comment on «On the quantum theory of molecules»." The Journal of Chemical Physics, 140: 037101.

Sutcliffe, B. T. and Woolley, R. G. (2021). "Is chemistry really founded in quantum mechanics?" Forthcoming in O. Lombardi, S. Fortin, and J. C. Martínez González (eds.), Quantum Chemistry: Philosophical Perspectives in Modern Chemistry. BerlinHeidelberg: Springer.

Trost, J. and Hornberger, K. (2009). "Hund's paradox and the collisional stabilization of chiral molecules." Physical Review Letters, 103: 023202.

Woolley, R. G. (1976). “Quantum theory and molecular structure.” Advances in Physics, 25: 27-52.

Woolley, R. G. (1978). "Must a molecule have a shape?" Journal of the American Chemical Society, 100: 1073-1078.

Woolley, R. G. (1982). "Natural optical activity and the molecular hypothesis.” Structure and Bonding, 52: 1-35.

Woolley, R. G. (1998). “Is there a quantum definition of a molecule?". Journal of Mathematical Chemistry, 23: 3-12.

Woolley, R. G. and Sutcliffe, B. T. (1977). "Molecular structure and the Born-Oppenheimer approximation." Chemical Physics Letters, 45: 393-398.

Zeh, H.-D. (2003). "Basic concepts and their interpretation.” Pp. 7-40 in E. Joos, H.-D. Zeh, C. Kiefer, D. Giulini, J. Kupsch, and I.-O. Stamatescu, Decoherence and the Appearance of a Classical World in Quantum Theory. Berlin: Springer.

Zurek, W. H., 1981, "Pointer basis of quantum apparatus: Into what mixture does the wave packet collapse?" Physical Review D, 24: 1516-1525.

Zurek, W. H. 1991, "Decoherence and the transition from quantum to classical." Physics Today, 44: 36-44. 